\title{
Rapid stereology based quantitative immunohistochemistry of dendritic cells in lymph nodes: A methodological study
}

Yvette van Hensbergen ${ }^{\text {a }}$,

Sylvia A. Luykx-de Bakker ${ }^{a}$,

Daniëlle A.M. Heideman a , Gerrit A. Meijer ${ }^{\text {b }}$, Herbert M. Pinedo ${ }^{a}$ and Paul J. van Diest ${ }^{b, *}$

${ }^{a}$ Department of Medical Oncology, Free University Hospital, Amsterdam, The Netherlands

${ }^{\mathrm{b}}$ Department of Pathology, Free University Hospital, Amsterdam, The Netherlands

Received 10 February 2000

Accepted 20 February 2001

This study was done to arrive at a fast and reliable protocol for assessment of fractional volumes of immunohistochemically stained dendritic cells in lymph nodes. Twenty axillary lymph nodes of patients with locally advanced breast cancer were immuno-histochemically stained with an S100 antibody. Fractional volumes of dendritic cells were assessed by stereology based quantitative immunohistochemistry using an interactive video overlay system including an automated microscope. The gold standard percentage of dendritic cells was the fractional volume of S100 stained cells in 500 fields systematically spread over the whole lymph node. Then, in a computer simulation, different sample sizes (1-200 fields of vision) were tested and the coefficient of variation (CV) for each sample size was calculated. The CV dropped with increasing sample size. A sample size of 100 fields of vision appeared to be optimal. Intra- and interobserver reproducibility appeared to be good (correlation coefficients of 0.95 and 0.86 , respectively) when re-analyzing the cases with the established protocol.

In conclusion, a fast and reliable assessment of the fractional volume of dendritic cells in lymph nodes is possible with semi-automated quantitative immuno-histochemistry. This method will form the base for further clinical studies

\footnotetext{
*Corresponding author: Paul J. van Diest, MD, PhD, Department of Pathology, Free University Hospital, PO Box 7057, 1007 MB Amsterdam, The Netherlands. Tel.: +31 20444 4080/4017; Fax: +31 20 444 2964; E-mail: pj.vandiest@azvu.nl.
}

into the immunological response in lymph nodes of patients with locally advanced breast cancer.

Keywords: Sampling, stereology, reproducibility, dendritic cells, S100, lymph nodes

\section{Introduction}

There is a growing interest in the role of dendritic cells (DCs) in the treatment of cancer $[4,5,16]$. DCs are bone marrow derived professional antigen presenting cells. The main function of DCs is to act as sentinels between the outside world and the body. DC progenitors are spread through the blood into peripheral tissues and after antigen uptake they migrate rapidly to $\mathrm{T}$ cell areas in the lymph nodes where they can initiate an immune response. In cancer patients an impaired cytotoxic $\mathrm{T}$ cell function contributes to the uncontrolled growth of tumor cells. In breast cancer patients a decreased number and function of DCs has been demonstrated [9]. Release of immunosuppressive agents by the primary tumor might be responsible for this DC disfunction $[10,14]$.

Recently we showed the results of neo-adjuvant chemotherapy plus granulocyte-macrophage colony stimulating factor (GM-CSF) in locally advanced breast cancer (LABC) [12]. We hypothesized that the encouraging survival data were not related to the chemotherapy results alone, but also the long-term administration of GM-CSF while the primary tumor and axillary lymph nodes were still in situ. An immunopotentiating effect might be the result of DC activation by GMCSF. A decrease in immunosuppressive agents, produced by the primary tumor, could contribute to this feature, while most patients showed an impressive clinical response. Chemotherapy induced shrinkage of the primary tumor is associated with release of tumor antigens, after tumor antigen uptake DCs migrate to the draining lymph nodes. 
To test our hypothesis, we needed to quantify the DCs in the lymph nodes. However, since the changes in the number of DCs may be small compared to the normal situation, reliable assessment of the numbers of DCs is required. Counting of all cells is hardly feasible, so an efficient sampling method will be necessary. Recently, it has been suggested that sampling based on stereological principles may provide an efficient and reliable approach for quantitative immunohistochemistry [6]. Therefore, we first undertook a methodological study evaluating the sample size and sampling procedure to arrive at a reproducible protocol to assess the fractional volume of DCs in the lymph nodes before performing a clinical study. The aim of the study was to establish a rapid and reproducible point counting method to assess fractional volume of S100 positive cells given an immunohistochemically stained section from a tissue block, and not to evaluate the contribution of variation at all different levels of the multistage sampling procedure.

\section{Material and methods}

\subsection{Sampling strategy}

Basically, 6 stages of selection can be discerned in the sampling procedure that must be considered for unbiased sampling: patients, lymph nodes from these patients, blocks from lymph nodes, sections from blocks, fields of vision (FOV) within sections, and points within fields of vision. The patients concerned representative LABC (stage IIIA or stage IIIB), that underwent neo-adjuvant treatment with a combination of high dose adriamycine and cyclophosphamide supported by GM-CSF as described before [13]. All lymph nodes of all patients were completely embedded without cutting them. Some non-randomness may have occurred here as cylindrical lymph nodes may have been embedded preferentially along their longest axis. From each block of all lymph nodes, one section was S100 immunohistochemically stained (see below). From these, 20 sections were selected covering the spectrum of fractional volumes of S100 cells based on subjective impression of the fractional volume: 6 nodes with a low amount $(\leqslant 10 \%), 7$ nodes with an intermediate amount $(>10 \%$ and $<20 \%)$ and 7 nodes with a high amount of S100 positive cells $(\geqslant 20 \%)$. Within the sections, FOV were sampled (systematically) at random (see below), so that all grid points were randomly positioned.

\subsection{Immunohistochemical staining}

The axillary lymph nodes had been fixed in neutral buffered formaldehyde and processed to paraffin according to standard procedures. From the paraffin blocks, $4 \mu \mathrm{m}$ thick sections (one from each lymph node) were cut and mounted on poly-l-lysine coated slides. Staining of the dendritic cells was performed with a rabbit polyclonal anti-S100 antibody (Dakopatts, Denmark). Endogenous peroxidase was blocked with $0.3 \% \mathrm{H}_{2} \mathrm{O}_{2}$ in methanol according to the Ventana protocol [17]. Enzymatic antigen retrieval was performed with protease II for 16 minutes at $40^{\circ} \mathrm{C}$ according to the Ventana protocol [17]. Staining of the slides with anti-S100 was also performed according to the Ventana protocol [17]. Briefly, all steps were performed at $40^{\circ} \mathrm{C}$. Incubation with the primary antibody was done for 20 minutes in a $1: 400$ dilution. For detection, the slides were incubated with a biotin-labeled secondary antibody, followed by incubation with peroxidase-labeled streptavidin. Staining was visualized with $0.05 \% 3,3^{\prime}$-diaminobenzidine tetrahydrochloride dihydrate containing $0.02 \% \mathrm{H}_{2} \mathrm{O}_{2}$, followed by an enhancement step with copper. Nuclear counter staining of the slides was done with hematoxylin. The slides were dehydrated in alcohol and mounted in DePeX mounting medium (BDH Laboratory Supplies, England). In the negative controls, the primary antibody was omitted. Normal tonsil was used as a positive control. Figure 1 shows some examples of the staining results.

\subsection{Quantitative immunohistochemistry}

Quantitative immunohistochemistry was performed using the QPRODIT interactive video overlay system (Leica, Cambridge, UK), consisting of a Pentium processor based PC with a computer mouse and a video overlay board linked to a CCD color camera mounted on a standard microscope equipped with an automated scanning stage and autofocus (PRIOR Scientific Instruments LTD, Cambridge, UK). This system enabled software driven selection of fields of vision and autofocussing. The live microscope image was displayed on the monitor of the PC in full color. Using a $\times 100 \mathrm{ob}-$ jective, the final on screen magnification was approximately $\times 3100$.

To establish the "gold standard" percentage of S100 positive cells, the contour of the lymph node was marked at low magnification and 500 fields systematically (equidistantly) spread over the whole marked 
(a)

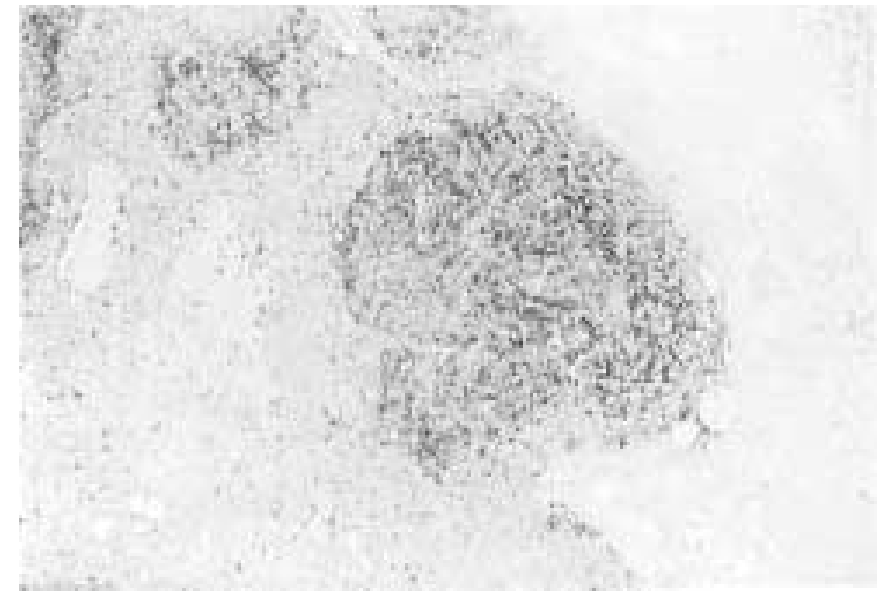

(b)

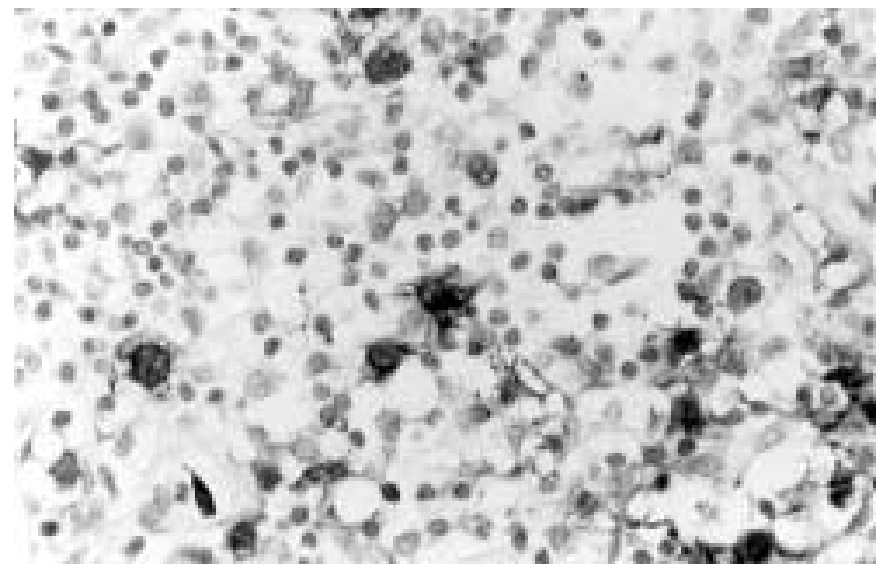

Fig. 1. Immunohistochemical staining performed with an S100 antibody on an axillary lymph node of a patient with locally advanced breast cancer. The large dark stained cells represent S100 positive cells. Figure 1(a) shows a lymph node stained for S100 in low magnification. Figure 1(b) shows a high magnification of the same lymph node.

lymph node were selected by means of the software driven scanning stage. At higher magnification, in each FOV, the points of a 6 points Weibel grid, electronically overlaid on the microscopic image on the screen (Fig. 2), were registered by the observer as overlying either S100 positive or negative cells. Based on the cumulative data of all fields of vision per lymph node, the gold standard fractional volume of dendritic cells was calculated.

To determine how the sample size (the number of fields assessed) affected the measurement precision, we performed a virtual systematic sampling experiment by computer simulation in the following way. From the 500 FOV available, varying numbers of systematic subsets of fields of vision were chosen. For example, when using a sample size of 1 FOV, 500 different samples are available. The variation between these different samples can be calculated by taking the mean of the percentages in each subset, calculating the standard deviation (sd), and subsequently calculating the coefficient of variation $(\mathrm{CV})$ by sd $\times 100 \% /$ mean. The higher the $\mathrm{CV}$, the greater the variation between samples and the lower the representativeness of an individual sample. When using a sample size of 2, 250 different systematic subsets are possible: a sample composed of fields no. 1 and 251, a sample of fields no. 2 and 252, etc., up to fields no. 250 and 500. Again, the $\mathrm{CV}$ over these 250 samples can be calculated. When using a sample size of 3, 165 different systematic samples are possible: a sample composed of fields no. 1, 167 and 333, a sample of fields no. 2, 168 and 334, etc., up to fields no. 165, 332 and 500. This was done up to a sample size of 200 fields of vision, and for each sample size, the $\mathrm{CV}$ was calculated. 


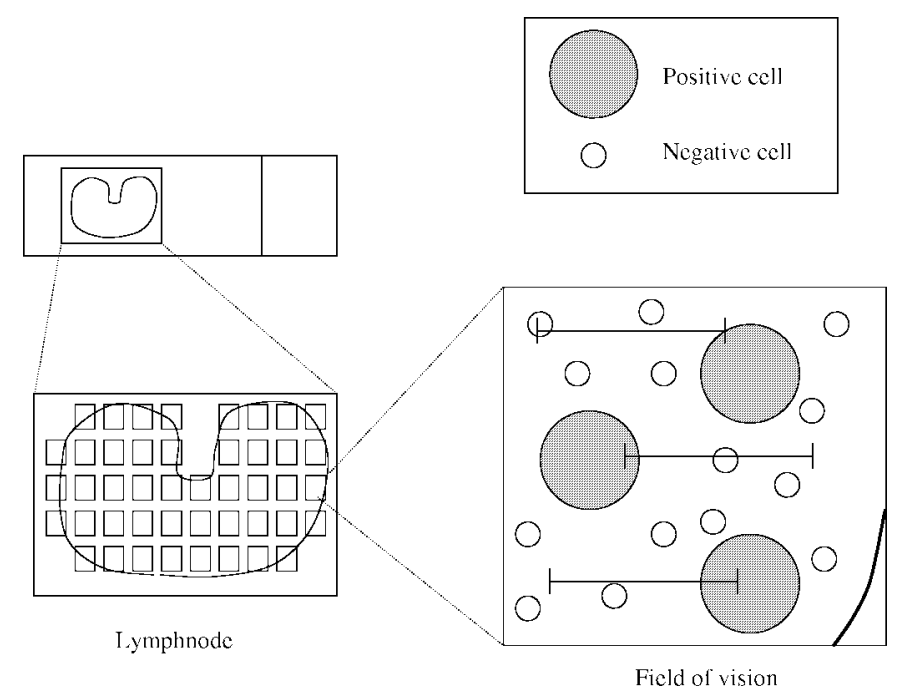

Fig. 2. Schematic illustration of the systemic random sampling method used to develop a protocol for interactive stereological immunoscoring with a cytoplasmatic marker. Fields of vision are systematically distributed throughout the entire lymph node. A six points Weibel-grid was used to count the positive cells in each field of vision.

Subsequently, the CVs were averaged for the 20 cases by taking the square root of the sum of individual values of $\mathrm{CV}^{2}$ divided by the number of $\mathrm{CVs}$, and this averaged $C V$ was plotted as a function of the sample size. The CV was expected to drop with increasing sample size. Based on this graph, the optimal sample size, defined as the minimum sample size which allowed to arrive at a desired $\mathrm{CV}$ of $\approx 15 \%$, could be determined.

The resulting measurement protocol including the set number of fields of vision to be analyzed based on the systematic sampling experiment described above was then tested for intra- and interobserver reproducibility. To assess intra-observer reproducibility, one observer $(\mathrm{YvH})$ measured the 20 specimens in a blind test twice with an interval of approximately 6 weeks, and two observers ( $\mathrm{YvH}$ and $\mathrm{DH})$ measured the 20 specimens independently to determine the interobserver reproducibility. Reproducibility was evaluated by linear regression analysis, calculating the correlation coefficient $R$, as well as the slope of the regression line and the intercept at the $y$-axis to detect systematic differences.

\section{Results}

In Table 1 the $\mathrm{CV}$ for each sample size set is shown for the first 5 specimens and the mean $\mathrm{CV} \pm$ sd calculated from the total 20 specimens. Figure 3 presents the mean $\mathrm{CV}$ as a function of the number of FOV mea- sured. Up to $75 \mathrm{FOV}$, the CV remained $>20 \%$ and substantial variation in $\mathrm{CV}$ was seen between the different specimens. From 100 FOV onwards, the CV dropped to $\approx 15 \%$ or lower and the variation of the CV between the specimens became less. At a sample size of 175 fields, the CV (\%) dropped to $10 \%$ and minimal CV variation between the specimens was seen. Considering the time spent on the measurements, a sample size of 100 fields of vision was considered to be adequate which yielded a CV of 16.1 .

Using this sample size of $100 \mathrm{FOV}$, intra- and interobserver reproducibility was good with correlation coefficients of 0.95 and 0.86 , respectively, without systematic differences (Figs 4 and 5).

\section{Discussion}

The aim of the study was to establish a rapid and reproducible point counting method to assess fractional volume of S100 positive cells given an immunohistochemically stained section from a tissue block, as this is the usual situation in daily practice, and not to evaluate the contribution of variation at all different levels of the multistage sampling procedure. The sampling strategy imposed some limitations, as there are (assuming the patients are representative) 5 stages of selection and have not been able to completely control sampling on all levels. All lymph nodes of all patients were completely embedded without cutting them, so that no prior selection of lymph nodes had taken place, 
Table 1

$\mathrm{CV}(\%)$ of assessments of fractional volume of $\mathrm{S} 100$ positive cells as a function of the number of fields of vision (\#FOV) assessed in a computer simulated systematic at random sampling experiment. Full data are shown of the first five cases as well as the mean of all 20 cases

\begin{tabular}{rrrrrrc}
\hline \#FOV $\backslash$ case & \multicolumn{1}{c}{1} & \multicolumn{1}{c}{2} & \multicolumn{1}{c}{3} & \multicolumn{1}{c}{5} & \multicolumn{2}{c}{ Mean of all cases } \\
& & & & & & $\sqrt{\sum \mathrm{CV}^{2} / n}$ \\
\hline 1 & 255.6 & 403.2 & 204.0 & 246.1 & 137.8 & 299.95 \\
2 & 157.5 & 161.4 & 145.9 & 177.1 & 97.9 & 204.20 \\
5 & 89.6 & 101.0 & 94.6 & 96.0 & 64.7 & 127.88 \\
10 & 87.8 & 77.9 & 81.9 & 93.5 & 39.7 & 91.60 \\
15 & 53.1 & 54.9 & 52.0 & 62.9 & 36.0 & 69.82 \\
20 & 61.5 & 53.2 & 66.4 & 60.7 & 29.4 & 57.28 \\
25 & 47.1 & 38.7 & 47.1 & 39.9 & 33.4 & 49.66 \\
50 & 37.6 & 24.3 & 39.8 & 15.2 & 24.3 & 30.11 \\
75 & 16.0 & 17.7 & 27.1 & 14.7 & 12.2 & 29.09 \\
100 & 9.5 & 27.1 & 36.5 & 9.1 & 13.9 & 16.08 \\
125 & 10.6 & 22.7 & 19.4 & 9.1 & 4.4 & 23.64 \\
175 & 8.4 & 17.5 & 18.3 & 4.1 & 4.2 & 10.99 \\
200 & 5.6 & 12.8 & 11.1 & 3.6 & 4.4 & 7.96 \\
\hline
\end{tabular}

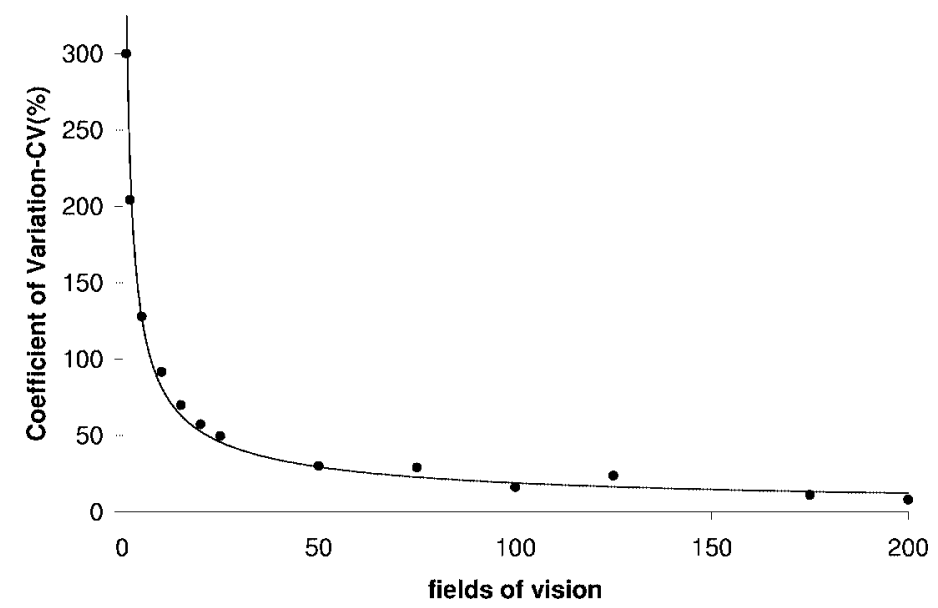

Fig. 3. Mean CV for the 20 specimens as a function of the number of fields of vision (FOV) used to assess the fractional volume of S100 positive cells in a specimen. Note that the CV gradually drops with increasing sample size.

and no selection within the nodes has taken place. Nonrandomness may have occurred for cylindrical nodes as they may have been embedded preferentially along their longest axis. However, while doing so, they can rotate around their longest axis introducing again an element of randomness. This cannot be avoided when working with archival material. All lymph nodes for all patients were S100 stained, so that no selection took place. Form each block, one section was made, and since the embedding can be regarded as "almost" random, the section taken is almost random as well. The 20 lymph nodes were selected from a total of about hundreds of lymph nodes, from which, as described, we picked 20 nodes covering the spectrum of fractional volumes of S100 cells based on subjective impression. Although this is not completely at random, there was no selection within the groups of low, intermediate and high fractional volumes, and this can be regarded as "almost" random. Within the sections, FOV were sampled (systematically) at random, so that grid points were randomly positioned. Altogether, despite some minor limitations in the sampling procedure, a high degree of randomness was present, to the extent that can be reached when working with archival material, and there are no indications that these minor limitations may have introduced relevant bias. 


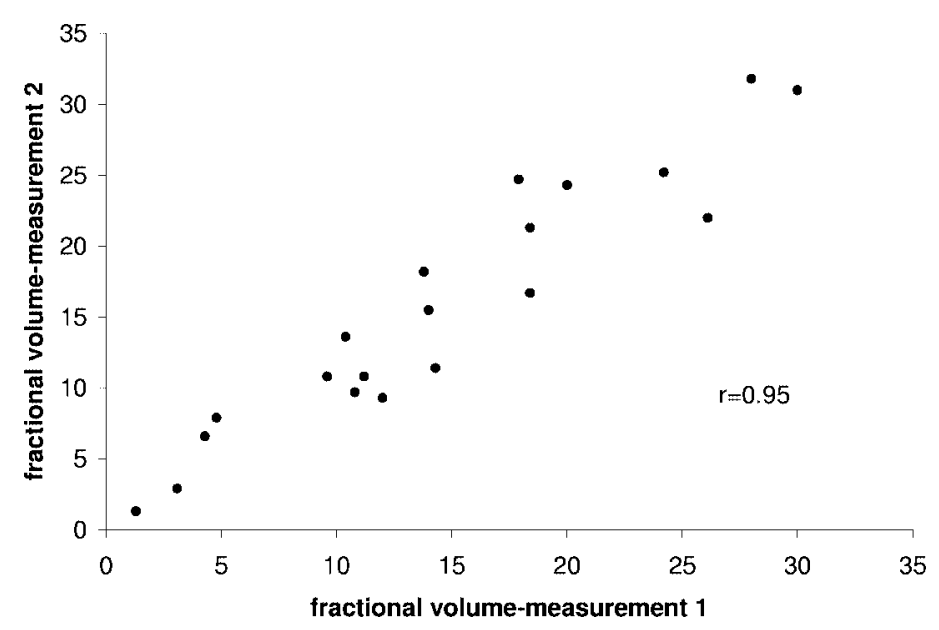

Fig. 4. Intra-observer reproducibility: results of duplicate assessment of fractional volume of S100 positive cells in 20 lymph node specimens.

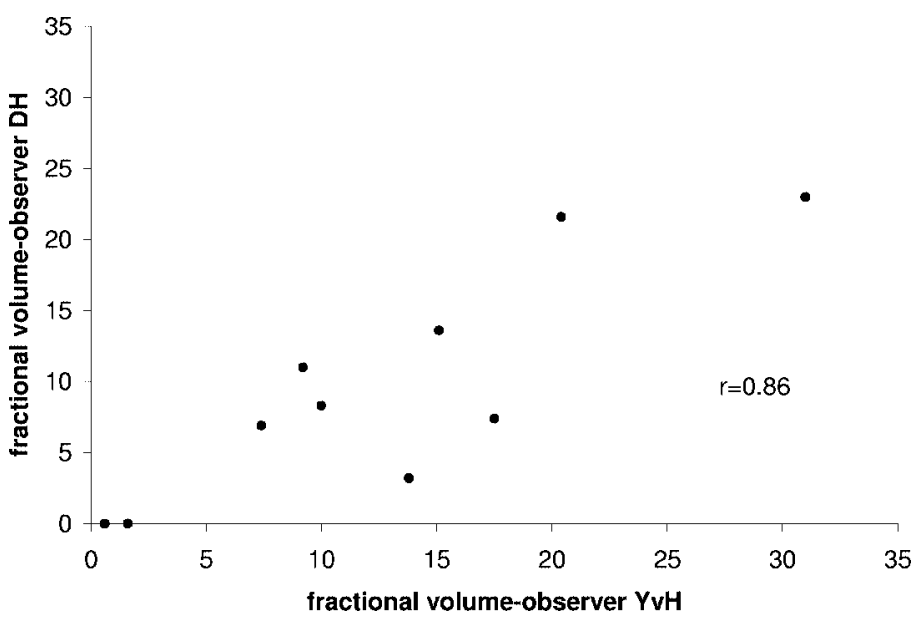

Fig. 5. Inter-observer reproducibility: assessment of fractional volume of S100 positive cells in 10 lymph node specimens measured independently by two observers.

Focussing on the level of sampling FOV in a given section, distribution of the sample FOV in the measurement field is an important issue. The sample spread of a defined sample size usually determines to a large extent the reproducibility of the measurement $[1,2,7,8,11]$. This is due to clustering of objects with similar characteristics. This can best be dealt with by spreading FOV systematically over the measurement field $[7,8]$. If the first FOV is chosen at random, all fields are random, so that a systematic at random approach is obtained. Since the sample spread is of greater influence than the number of FOV studied and the number of observations per FOV, measurement procedures can be made more efficient by taking fewer measurements in more FOV that are spread systematically at random over the predefined measurement area, the "do more less well" prin- ciple [11]. Systematic random sampling was demonstrated to be the most reproducible method for different kinds of morphometric measurements [1,3,6-8]. It has also been shown that a similar approach is useful for assessment of the number of immunohistochemically positively stained nuclei [15]. We now show that this approach is also useful for fast and reliable assessment of cytoplasmic immunohistochemical staining. Apart from S100, other cytoplasmic antigens can be quantitated in a similar way.

In this study we used the anti-S100 antibody to stain the dendritic cells in the lymph nodes. Although this polyclonal antibody is not fully specific for dendritic cells, it was possible to separate the dendritic cells from the other cells based on their characteristic den- 
dritic morphology that was well visible at the high magnification used for measurement.

A systematic random approach was shown to be necessary for assessment of fractional volume of S100 positive cells since they also tend to cluster. Especially on the borders of the lymph nodes clusters of S100 positive cells are seen (Fig. 1(a)). It appeared to be necessary to measure about 100 fields of vision systematically at random spread over the lymph node, which allowed achieving a theoretical CV of $<15 \%$. This takes approximately 5-8 minutes, which can be achieved by the high degree of automation due to the scanning stage and autofocus. Obviously, similar assessments can be done without such automatic devices, but it will take more time. Intra- and interobserver reproducibility of the resulting protocol was high. Although increasing the number of FOV measured in a specimen resulted in even better CVs and less variation between the specimens, this is more time consuming. Therefore a protocol based on 100 fields of vision seems to be the best compromise between precision and workload. Stereology based semi-automatic quantitative immunohistochemistry is in general interesting for quantitation of different nuclear and cytoplasmic antigens due to the cheap equipment, high speed, high accuracy and good reproducibility.

In conclusion, fast and reliable assessment of the fractional volume of dendritic cells in lymph nodes is possible with semi-automated quantitative immunohistochemistry based on systematic at random sampling. This method will form the base for further clinical studies into the immunological response in lymph nodes of patients with locally advanced breast cancer, and is also applicable to quantitation of other cytoplasmic antigens. Preliminary results indicate that the suggested protocol to assess dendritic cells yields clinically relevant results in patients with locally advanced breast cancer [18].

\section{References}

[1] M. Brinkhuis, G.A. Meijer, J.A.M. Beliën, P.J. van Diest and J.P.A. Baak, Volume-weighted mean nuclear volume and nuclear area in advanced ovarian cancer: An investigation of sampling methods, sample size and reproducibility, Anal. Quant. Cytol. Histol. 97 (1995), 284-290.

[2] J. Brugghe, J.P.A. Baak, G.A. Meijer, P.J. van Diest and M. Brinkhuis, Rapid and reliable assessment of volume percentage of epithelium in borderline and invasive ovarian tumors, Analyt. Quant. Cytol. Histol. 20 (1998), 14-20.

[3] W.G. Cochran, Sampling Techniques, Whiley, New York, 1977.

[4] C. Codon, S.C. Watkins, C.M. Celluzzi, K. Thompson and L.D. Falo, Jr., DNA-based immunization by in vivo transfection of dendritic cells, Nature Medicine 2 (1996), 1122-1128.
[5] E. Coveney, G.H. Wheatley III and H.K. Lyerly, Active immunization using dendritic cells mixed with tumor cells inhibits the growth of primary breast cancer, Surgery 122 (1997), 228-234.

[6] P.J. van Diest, P. van Dam, S.C. Henzen-Logmans, E. Berns, M.E.L. van der Burg, J. Green and I. Vergote, A scoring system for immunohistochemical staining: consensus report of the task force for basic research of the EORTC-GCCG, J. Clin. Pathol. 50 (1997), 801-804.

[7] J.C. Fleege, P.J. van Diest and J.P.A. Baak, Systemic random sampling for selective interactive nuclear morphometry in breast cancer sections; Refinement and multiobserver evaluation, Anal. Quant. Cytol. Histol. 15 (1993), 281-289.

[8] J.C. Fleege, P.J. van Diest and J.P.A. Baak, Computer assisted efficiency testing of different sampling methods for selective nuclear graphic tablet morphometry, Lab. Invest. 83 (1990), 270-275.

[9] D.I. Gabrilovich, J. Corak, I.F. Ciernik, D. Kavanaugh and D.P. Carbone, Decreased antigen presentation by dendritic cells in patients with breast cance, Clin. Cancer Res. 3 (1997), 483-490.

[10] D.I. Gabrilovich, H.L. Chen, K.R. Girgis, H.T. Cunningham, G.M. Meny, S. Nadaf, D. Kavanaugh and D.P. Carbone, Production of vascular endothelial growth factor by human tumors inhibits the functional maturation of dendritic cells, $\mathrm{Na}$ ture Medicine 2 (1996), 1096-1103.

[11] H.J.G. Gundersen and R. Osterby, Optimizing sampling efficiency of stereological studies in biology: or "Do more less well!", J. Microscopy 121 (1981), 65-73.

[12] A.H.H. Honkoop, S.A. Luykx-de Bakker, K. Hoekman et al., Dose-intensive neo-adjuvant chemotherapy with GM-CSF in locally advanced breast cancer: a chemo-immunotherapeutic approach, Unpublished (Submitted), Free University Hospital, Amsterdam, The Netherlands.

[13] A.H.H. Honkoop, P.J. van Diest, J.S. de Jong, S.C. Linn, K. Hoekman, J. Wagstaff and H.M. Pinedo, Prognostic role of clinical, pathological and biological characteristics in patients with locally advanced breast cancer, Br. J. Cancer 77 (1998), 621-626.

[14] A. Morel, S. Quarantino, D.C. Douek and M. Londei, Split activity of interleukin-10 on antigen capture and antigen presentation by human dendritic cells: definition of a maturative step, Eur. J. Immunol. 27 (1997), 26-34.

[15] W. Polkowski, G.A. Meijer, J.P. Baak, F.J. ten Kate, H. Obertop, G.J. Offerhaus and J.J. van Lanschot, Reproducibility of p53 and Ki-67 immunoquantitation in Barrett's esophagus, $A n$ alyt. Quant. Cytol. Histol. 19 (1997), 246-254.

[16] E.M. Sotomayor, I. Borello and H.I. Levitsky, Tolerance and cancer: a critical issue in tumor immunology, Crit. Rev. Oncol. 7 (1996), 433-456.

[17] Inc Ventana ES, Ventana Medical Systems, Operators Manual, 1994.

[18] S.A. Luykx-de Bakker, T.G. de Gruyl, P.J. van Diest and H.M. Pinedo, Draining lymph node dendritic cells after neo-adjuvant chemotherapy plus GM-CSF in locally advanced breast cancer: an immunhistochemical study, J. Leukocyte Biol. 64 (Suppl. 2) (1998), 97. 


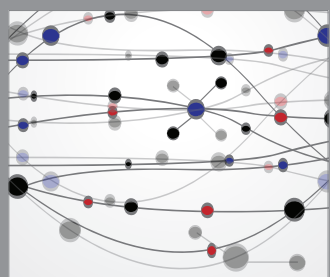

The Scientific World Journal
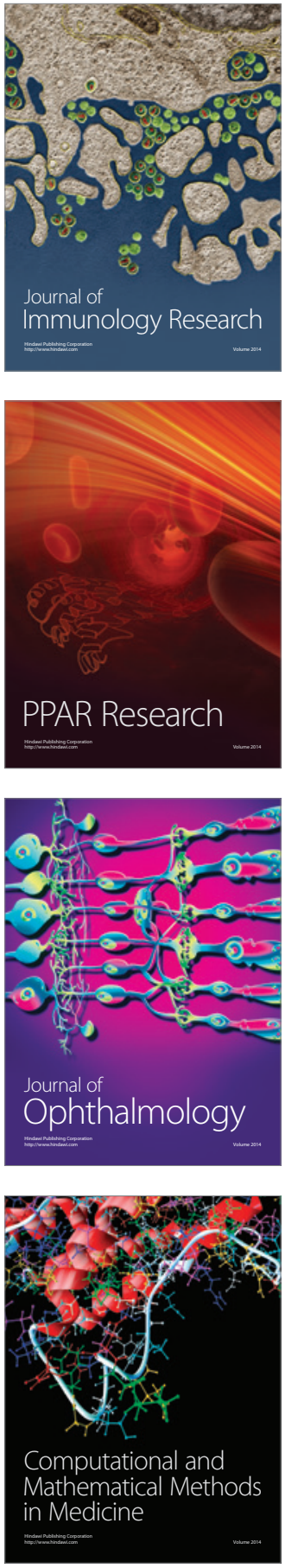

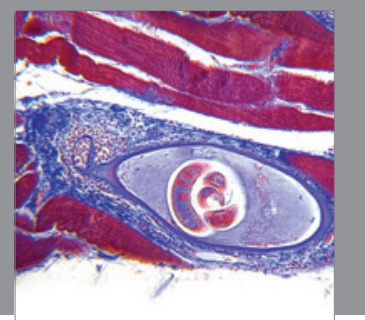

Gastroenterology

Research and Practice
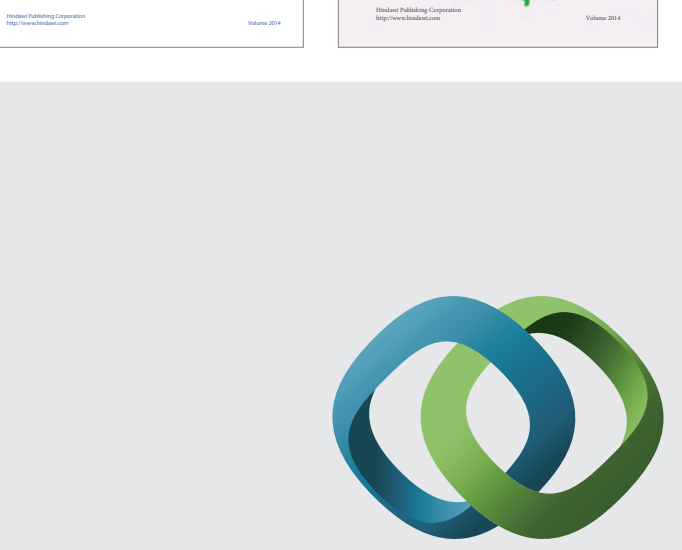

\section{Hindawi}

Submit your manuscripts at

http://www.hindawi.com
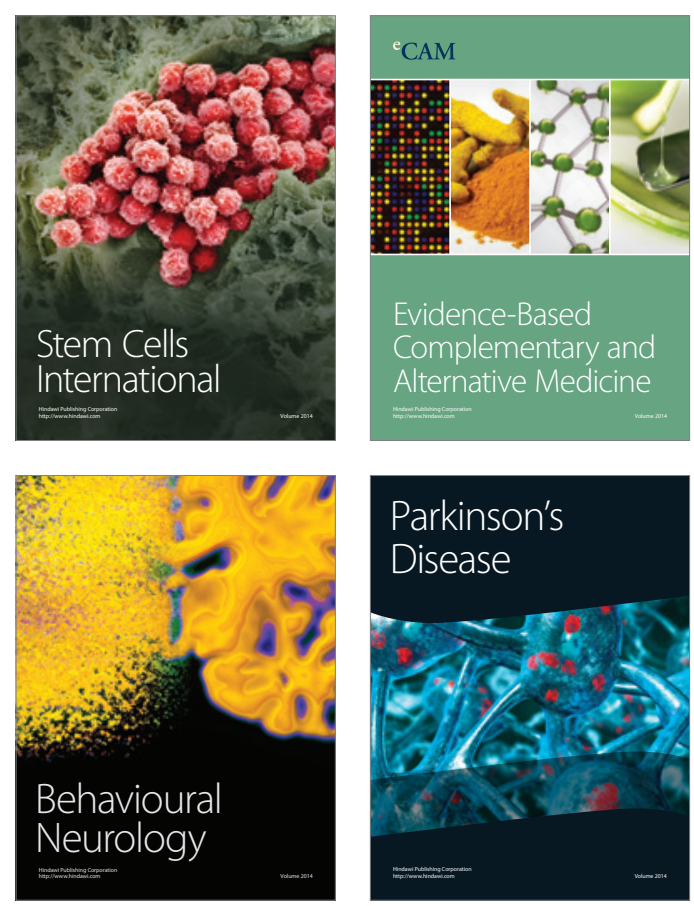

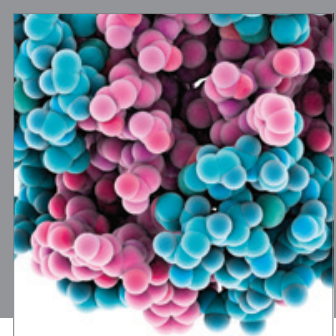

Journal of
Diabetes Research

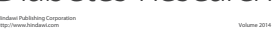

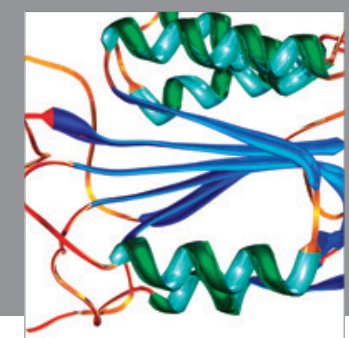

Disease Markers
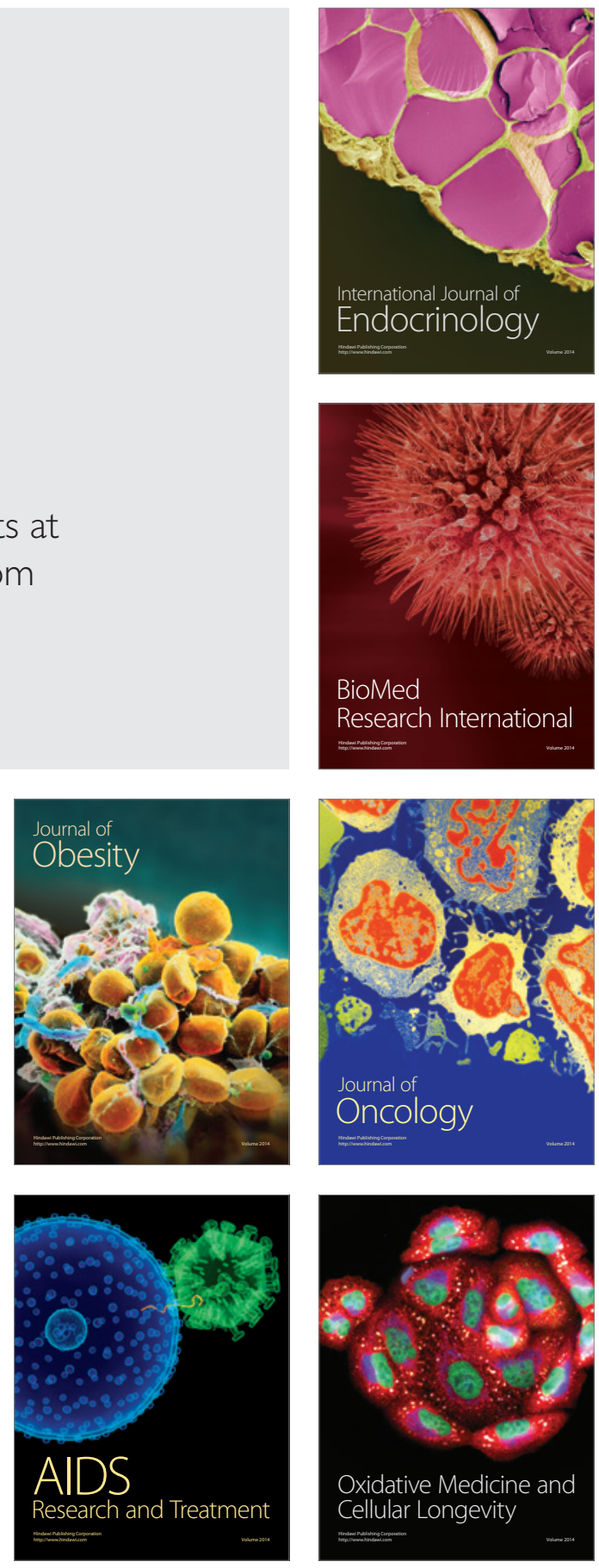